\title{
Imaging of Postoperative Avascular Necrosis of the Ankle and Foot
}

\author{
Craig A. Buchan, B.Com., M.B.B.S., F.R.A.N.Z.C.R. ${ }^{1,2,3}$ Dawn H. Pearce, M.D., F.R.C.P.C. ${ }^{4}$ \\ Johnny Lau, M.D., F.R.C.S.C. ${ }^{5}$ Lawrence M. White, M.D., F.R.C.P.C. ${ }^{1,2}$ \\ ${ }^{1}$ Joint Department of Medical Imaging,Mount Sinai Hospital, Toronto, \\ Ontario, Canada \\ 2 University Health Network and Women's College Hospital, University \\ Address for correspondence and reprint requests Dawn $\mathrm{H}$. Pearce, \\ Department of Radiology, St. Michael's Hospital, 30 Bond St., Toronto, \\ of Toronto, Toronto, Ontario, Canada \\ ${ }^{3}$ Department of Medical Imaging, Gold Coast Hospital, Southport, \\ Australia \\ ${ }^{4}$ Department of Radiology, St. Michael's Hospital, University of \\ Toronto, Toronto, Ontario, Canada \\ ${ }^{5}$ Department of Orthopedic Surgery, Toronto Western Hospital, \\ University Health Network and Women's College Hospital, University \\ of Toronto, Toronto, Ontario, Canada \\ Ontario, Canada M5B 1W8 (e-mail: pearced@smh.toronto.on.ca).
}

Semin Musculoskelet Radiol 2012;16:192-204.

\begin{abstract}
Keywords

- avascular necrosis

- osteonecrosis

- foot and ankle

- postoperative

- magnetic resonance imaging

Avascular necrosis (AVN) of the ankle and foot is an uncommon and often unexpected postoperative complication in patients with persistent pain and disability postprocedure. Artifacts from metallic implants may obscure characteristic imaging signs of AVN, and radiography and computer tomography are the mainstay imaging modalities of the postoperative ankle and foot. MRI and nuclear medicine imaging play an important complementary role in problem solving and excluding differential diagnostic considerations including infection, nonunion, occult fracture, and secondary osteoarthritis.

This review article evaluates different imaging modalities and discusses characteristic sites of AVN of the ankle and foot in the postoperative setting including the distal tibia, talus, navicular, and first and lesser metatarsals. Radiologists play a key role in the initial diagnosis of postoperative AVN and the surveillance of temporal evolution and complications including articular collapse and fragmentation, thus influencing orthopedic management.
\end{abstract}

The terms avascular necrosis (AVN) and osteonecrosis are used interchangeably in the medical literature and imply bone death secondary to circulatory disturbance. ${ }^{1}$ Disturbance of the vascular supply and subsequent deprivation of oxygen can result from traumatic or compressive arterial inflow disruption, intraluminal vascular occlusion, or venous outflow obstruction.

The most common sites of AVN in the body after traumatic disruption to the intramedullary blood supply are the femoral head, humeral head, scaphoid, and talus. ${ }^{1-3}$ Postoperative AVN is a well-recognized complication in the hip and also occurs rarely in the knee after arthroscopy; contributory technical factors include surgical disruption of extraosseous blood supply, hardware placement injury to nutrient arteries, and thermal necrosis. ${ }^{4-6}$ Subchondral and intramedullary hemorrhage at the time of initial injury or surgery may induce intravascular coagulation mediated by embolic lipid, hypofibrinolysis, proteolytic enzymes, and tissue factor release. Furthermore, deposition of fibroadipose granulation tissue adjacent to necrotic bone and persistent mechanical stress may impair the ingrowth of new vessels, thereby impairing healing. The common denominator in AVN is compromised blood flow to the affected area.

AVN has been described in almost every bone of the ankle and foot, with trauma the leading cause. ${ }^{3}$ The talus is most commonly affected due to its tenuous blood supply.,
Issue Theme Imaging of the Postoperative Ankle and Foot; Guest Editor, James M. Linklater, F.R.A.N.Z.C.R.
Copyright $\odot 2012$ by Thieme Medical Publishers, Inc., 333 Seventh Avenue, New York, NY 10001, USA. Tel: +1(212) 584-4662.
DOI http://dx.doi.org/ 10.1055/s-0032-1320060. ISSN $1089-7860$. 
Nontraumatic etiologies of AVN include corticosteroids, alcoholism, hyperlipidemia, hemoglobinopathies, inherited thrombophilias, renal transplantation, diabetes, systemic lupus erythematosus (SLE), and irradiation. ${ }^{2,3,7}$ Systemic causes including sickle cell disease, SLE, corticosteroids, and ischemia due to diabetes may cause multifocal infarctions of the foot and ankle and have a predilection for the talus and calcaneus. ${ }^{8}$ Some authors limit the definition of AVN to include only systemic causes, arguing that bone necrosis is a histological end point of many disease processes including severe osteoarthritis, fractures, infections, and tumors. ${ }^{9}$ In posttraumatic cases involving fractures of the distal tibia and talar neck, quantifying the effect of the initial traumatic disruption of blood supply as opposed to subsequent orthopedic intervention is difficult. A further complicating factor in assessing pathogenesis and determining the clinical prevalence is the observation that although most cases of posttraumatic AVN of the foot manifest clinically within the first 10 months after injury, cases in the distal tibial metaphysis may remain clinically asymptomatic. ${ }^{3}$ Our discussion is limited to include the characteristic patterns of bone necrosis observed in the postoperative setting. Purported so-called idiopathic or stress-related AVN of the foot bones including the sesamoid bones, Mueller-Weiss disease of the lateral navicular bone, and Freiberg's disease of the metatarsal heads are beyond the scope of this review article.

\section{Imaging Techniques}

\section{Radiographs}

The goal of imaging following open reduction and internal fixation or reconstructive surgery is the assessment of healing. In the first 4 months postsurgery, clinical distinction between AVN and normal postoperative findings is difficult, and serial radiographs are performed in the orthopedic outpatient setting in conjunction with clinical assessment. Routine radiographic evaluation of the ankle and foot should include as a minimum anteroposterior (AP) and oblique "mortise" views of the ankle, weightbearing AP, and oblique views of the foot and lateral weightbearing views of the ankle and foot. Coned radiographs centered on the head of the first metatarsal are useful if clinical suspicion of pathology. Comparison with the prior radiographs and the contralateral side is often helpful to observe early signs of AVN.

Detecting early stages of AVN is difficult radiographically and must be inferred by a lack of bone resorption relative to disuse osteopenia in the adjacent bones secondary to immobilization. ${ }^{8}$ The "Hawkins sign" in the talar dome, seen on AP radiographs as a thin subchondral radiolucent line 4 to 8 weeks after talar neck fracture/surgery, represents resorption of subchondral bone and is predictive of the absence of AVN (-Fig. 1). ${ }^{10}$ Absence of the Hawkins sign, however, does not predict the occurrence of AVN. ${ }^{11}$ Increased radiodensity, mild osseous resorption, and small subchondral cyst formation may be seen as part of the normal healing process after successful osteotomy. Subtle early signs of AVN may be obscured by soft tissue swelling, overlying plaster casts, skin dressings, and skin closure devices.

Later in the process, increased sclerosis of infarcted bone becomes more apparent as new bone is laid down over necrotic trabeculae. The infarcted region becomes sharply marginated due to revascularization and resorption of the edges, leaving a radiolucent rim around the devitalized bone. ${ }^{12}$ At this stage the "crescent sign," representing a linear subchondral fracture, may be seen in the talus and distal tibial plafond but is less reliably detectable in the small bones of the foot. End-stage radiographic findings of AVN, including collapse of the articular surface and the development of secondary osteoarthritis, may be the first observable radiographic abnormality in the metatarsals.

\section{Computed Tomography}

Multidetector computed tomography (CT) is a useful tool in the postoperative ankle and foot to evaluate the position of orthopedic hardware, presence or absence of bone bridging, differential density of infarcted bone, and assessment of articular surfaces for subtle depression, collapse, or fragmentation. CT is more sensitive than radiographs in detecting signs of AVN including marginated sclerosis, the Hawkins sign, and the crescent sign (-Fig. 1). Preoperative CT is often obtained in the polytrauma setting and in cases of complicated foot and ankle fractures, and we find temporal comparison useful in assessment for AVN.

Technique factors may be used to reduce beam hardening and subsequent reconstruction artifacts associated with $\mathrm{CT}$ in the presence of metallic hardware, thus improving diagnostic sensitivity. Traditional means include altering image acquisition parameters such as the peak voltage (increased $\mathrm{kVp}$ ), tube charge (increasing mAs or utilizing automatic dose control option), and use of narrow collimation settings. Image distortion at the bone/hardware interface ("splay artifact") previously seen with the imaging of large joint prostheses is less pronounced with the greater number of detector rows and lower pitch settings of modern machines. ${ }^{13}$ Use of smooth reconstruction filters and slices slightly thicker than the minimum slice thickness available on the scanner also reduces splay artifact and image noise. ${ }^{13,14}$ Modern scanners suppress streak artifacts caused by metal implants without increasing dose by applying inbuilt reconstruction techniques to smooth areas with deficient photon counts in the raw data. In the small joints of the ankle and foot where minimal hardware is usually present, artifacts are usually less pronounced (-Fig. 2). We routinely perform helical or volumetric $\mathrm{CT}$ examinations with a $120-\mathrm{kVp} / 200 \mathrm{mAs}$ technique with automatic dose control modulation using $0.5-\mathrm{mm}$-thick slices, reconstructed using bone sharp and soft tissue standard filters at $2 \mathrm{~mm}$ thickness by $2-\mathrm{mm}$ intervals in three planes.

\section{MRI}

MRI is the most sensitive imaging technique for detecting AVN in the early stages, with the initial finding of bone marrow edema (ill-defined hypointensity on T1-weighted images and patchy increased signal on T2-weighted/fluidsensitive sequences). Bone marrow edema correlates with histological findings of ischemic death of hematopoietic cells, capillary endothelial cells, and lipocytes with subsequent increase in extracellular fluid in the bone, present by the second week after infarction. ${ }^{3}$ In the traumatic or 

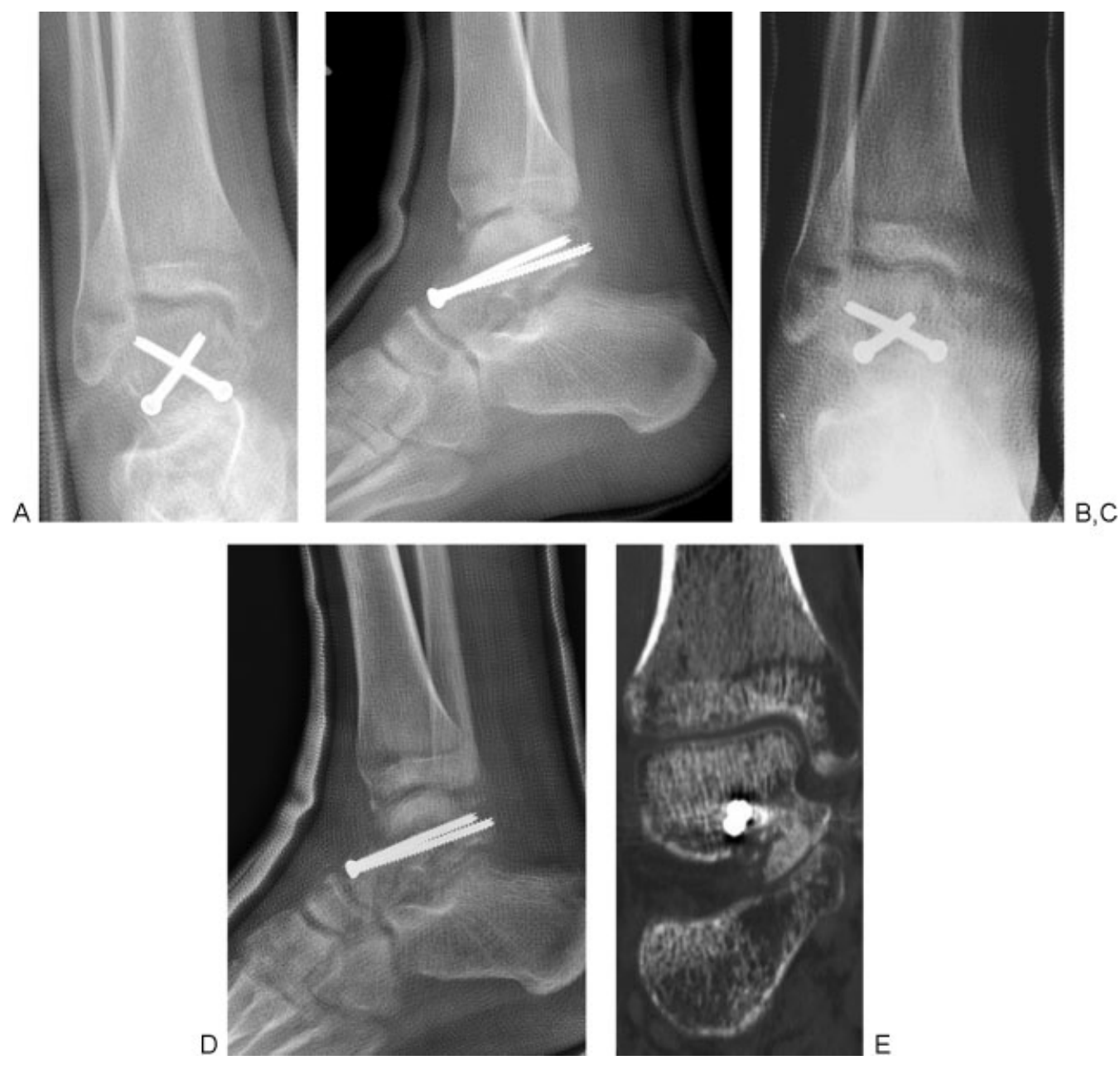

Figure 1 Evolving Hawkins sign after crossing interfragmentary screw fixation of minimally displaced talar neck fracture, indicating resorption of subchondral bone and the absence of avascular necrosis (AVN). (A, B) Anteroposterior (AP) and lateral radiographs 4 weeks after injury show subtle subchondral lucency partly obscured by overlying plaster. (C, D) AP and lateral radiographs at 10 weeks demonstrate marked diffuse osteopenia and prominent subchondral lucency, detectable despite the overlying plaster cast. (E) Noncontrast computed tomography with coronal reformats better depicts subchondral bone resorption and fracture healing. This patient did not subsequently develop AVN.

postoperative setting, marrow edema and hemorrhage also occurs due to disruption of the trabeculae and leakage of fluid and blood products into the extracellular space. ${ }^{15}$ Congestive marrow edema, proposed as a mechanism of AVN in patients with marrow packing disorders or on corticosteroids and predominantly involving the femoral head and proximal humerus, is likely secondary to vascular occlusion or thrombosis and is probably less important in the postoperative ankle and foot.

Marrow edema-like signal is a nonspecific MR finding that may be secondary to multiple causes such as hematopoietic marrow reconversion, infection, trauma, and tumor. ${ }^{16}$ Elias and colleagues described a bone marrow edema pattern in the foot and ankle seen within the first 12 weeks of immobilization for treatment of lower limb injury predominating in subchondral, subcortical, and subenthesial locations. This subsequently stabilized or resolved completely by 18 weeks and did not correlate with new pain or the clinical syndrome of reflex sympathetic dystrophy. ${ }^{17}$ In the postoperative setting, early bone marrow edema may be multifactorial and alone should be interpreted with caution or further characterized with follow-up imaging.

As radiographic sclerosis develops in the infarcted bone, there is corresponding low signal intensity on both T1- and T2-weighted images. The reparative interface between necrotic tissue and viable granulation tissue manifests as the "double-line sign," a low signal intensity rim in which the inner aspect becomes high signal on T2-weighted images. This sign is characteristic of AVN of the femur and humeral head but less frequently observed in the ankle and foot. Weishaupt et al suggested that the double-line sign is more commonly seen in systemic disorders that have a predilection for the talus and calcaneus. ${ }^{18}$ We have observed this sign in multiple cases of AVN of the distal tibia (-Fig. $\mathbf{3}$ ).

In addition to suggesting the diagnosis of AVN, MR imaging offers important information to the orthopedic surgeon including the site and size of the involved segment, presence of associated fractures, and the integrity of overlying articular cartilage and subchondral bone. These factors affect subsequent management decisions including whether to perform 


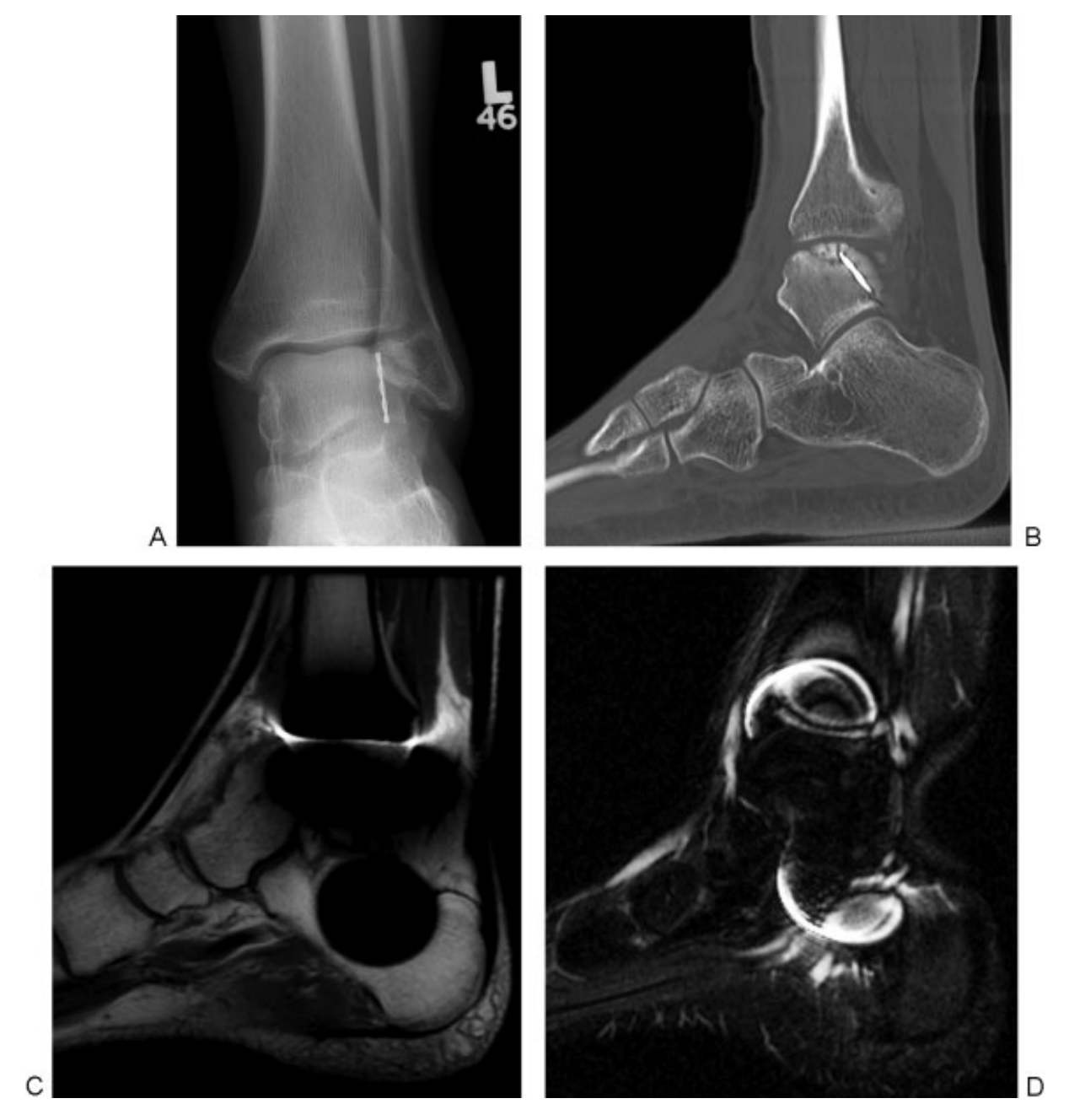

Figure 2 Comparison of computed tomography (CT) and MRI artifacts from fractured stainless-steel screw fragment in talar dome after microdrilling of osteochondral defect of talar dome. (A, B) Anteroposterior radiograph of the ankle and noncontrast CT with sagittal reformats provide high-resolution detail of the screw fragment and the sclerotic osteochondral lesion. (C, D) Sagittal fat saturation fast-spin echo T1-weighted and short tau inversion recovery MRI images are nondiagnostic due to extensive susceptibility and misregistration artifact.

core decompression, bone grafting, arthrodesis, or joint replacement. $^{19}$

The postsurgical MR protocols for imaging the foot and ankle at our institution are listed in - Tables $\mathbf{1 A}$ and 1B. We do not routinely administer intravenous gadolinium unless there is a high index of suspicion for infection complicating orthopedic hardware placement. Although intravenous gadolinium may aid in the assessment of the extent of nonenhancing infarcted bone for surgical planning, we do not usually find this necessary.

Artifacts caused by ferromagnetic screws and implants and metal shavings from the use of surgical instruments may be problematic in MR image acquisition and interpretation and are more severe if cobalt-chrome or stainless-steel alloy implants are used compared with titanium (-Fig. 2). The use of fast spin-echo and short tau inversion recovery sequences reduces metal-related artifacts compared with gradient-echo and frequency-selective fat saturation sequences. ${ }^{13,14}$ We have observed an increase in susceptibility and misregistration artifacts increase with greater field strength (1.5 T to $3.0 \mathrm{~T}$ ), although broader receiver bandwidth and the higher gradient field strengths used partially offsets the effect. Imaging pa- rameter optimization at higher field strength may include using a small field of view, increasing the resolution (matrix size), use of thin slices, increasing the echo train length, and increasing the bandwidth. ${ }^{14}$

\section{Nuclear Medicine}

Three phase Tc-99m-diphosphonate (Tc-99m-MDP) bone scintigraphy is not commonly used in clinical practice to confirm the diagnosis of AVN of the ankle and foot. Recent studies have demonstrated that bone scintigraphy is less sensitive than MRI in diagnosing symptomatic AVN, with MRI detecting $100 \%$ of lesions, whereas bone scanning detected $72 \%$ of oligofocal cases (two or fewer joints) and $45 \%$ of multifocal cases. ${ }^{20}$ Sensitivity is reduced in AVN cases in the ankle compared with the hip and knee. Three-phase bone scintigraphy findings in acute AVN include decreased or normal flow activity with decreased activity on delayed phases. The "bull's-eye sign" of a central region of photopenia with a surrounding rim of hyperemia is infrequently observed in the femoral head and rarely seen in the foot and ankle. $^{21}$ 

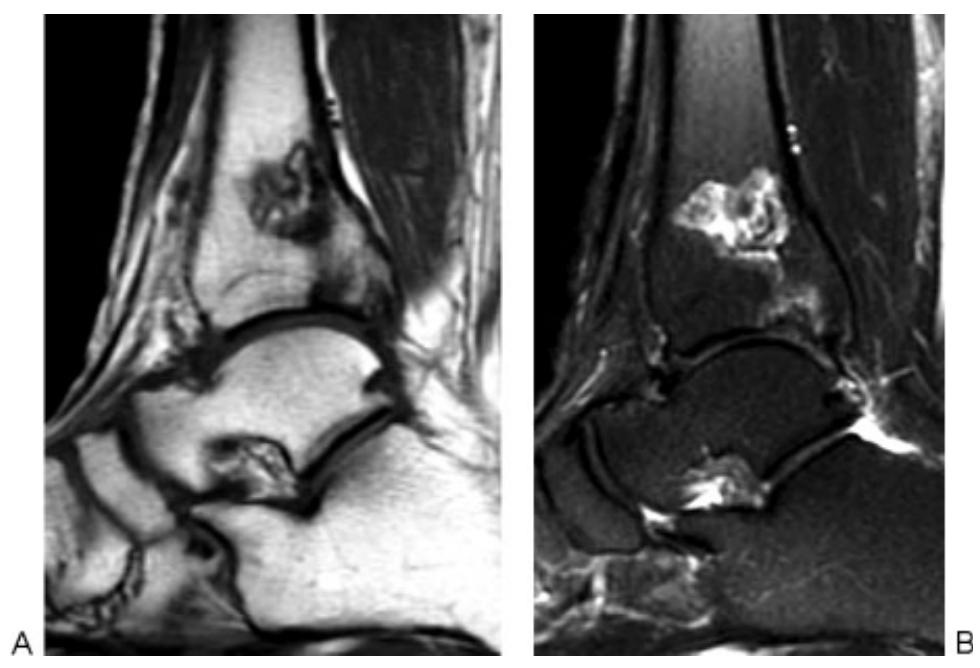

Figure 3 "Double-line sign" characteristic of avascular necrosis (AVN) in distal tibial metaphysis. Subsequent collapse of the articular surface necessitated tibio-talar arthrodesis in this patient. (A, B) Sagittal T1 fast spin-echo and fat saturation T2-weighted MRI, demonstrating serpiginous region of AVN/bone infarction with low signal intensity rim on $\mathrm{T} 1$ images. The inner aspect of the rim becomes high signal on T2-weighted images.

Tc-99m-MDP bone scintigraphy in combination with gallium scan or labeled white blood cell scan aids in excluding infection that may mimic AVN on other imaging modalities. Acute osteomyelitis shows tracer enhancement within 48 hours in all three phases on Tc-99m-MDP scans, although in chronic infection no increased flow is usually seen. Threephase bone scan accuracy is only 50 to $70 \%$ because postoperative and degenerative changes are difficult to distinguish.

Table 1A Imaging Parameters Ankle and Foot MRI with Metal Reduction - 1.5T

\begin{tabular}{|l|l|l|l|l|l|}
\hline Ankle (Supine Position) & T1 FSE & STIR & PD & T2 FS FSE & PD \\
\hline Plane & Sagittal & Sagittal & Coronal & Axial & Axial \\
\hline TR (ms) & 480 & 2000 & 2000 & 5600 & 4500 \\
\hline TE (ms) & 12 & 30 & 30 & 40 & 38 \\
\hline TI (ms) & & 150 & & 150 & \\
\hline FOV (mm) & $160 \times 160$ & $160 \times 160$ & $140 \times 140$ & $140 \times 140$ & $140 \times 140$ \\
\hline Matrix size (1.5 T) & $192 \times 256$ & $256 \times 256$ & $448 \times 328$ & $140 \times 320$ & $288 \times 512$ \\
\hline Thickness (mm)/Skip & $3 / 0$ & $3 / 0$ & $3 / 0$ & $3 / 0$ & $3 / 0$ \\
\hline NEX & 1 & 2 & 1 & 2 & 1 \\
\hline Turbo factor/ETL & 3 & 5 & 6 & 7 & 9 \\
\hline Bandwidth (Hz/pixel) & $250-395$ & 401 & 250 & 401 & 391 \\
\hline Forefoot (Supine Position) & T1 FSE & T2 FS & PD & T1 FSE & T2 FS \\
\hline Plane & Sagittal & Sagittal & Coronal & Axial & Axial \\
\hline TR (ms) & 480 & 3000 & 4400 & 470 & 5000 \\
\hline TE (ms) & 12 & $70-80$ & 40 & $10-14$ & $80-90$ \\
\hline FOV (mm) & $160 \times 160$ & $260 \times 260$ & $140 \times 140$ & $140 \times 140$ & $140 \times 140$ \\
\hline Matrix size (1.5 T) & $192 \times 256$ & $256 \times 256$ & $512 \times 256$ & $256 \times 256$ & $256 \times 192$ \\
\hline Thickness (mm) & $4 / 0$ & $4 / 0$ & $4 / 0$ & $3 / 0$ & $3 / 0$ \\
\hline NEX & 1 & 2 & 1 & 2 & 1 \\
\hline Turbo factor/ETL & 3 & 8 & 6 & 7 & 8 \\
\hline Bandwidth (Hz/pixel) & $250-395$ & $150-250$ & 250 & $250-395$ & $150-250$ \\
\hline
\end{tabular}

FSE, fast spin echo; STIR, short tau inversion recovery; FS, frequency-selective fat saturated; PD, proton density; FOV, field of view; NEX, number of excitations; ETL, echo train length; BW, bandwidth.

T2 FS is usually used when imaging the forefoot rather than STIR unless significant artifacts result. 
Table 1B Imaging Parameters Ankle and Foot MRI with Metal Reduction - 3T

\begin{tabular}{|l|l|l|l|l|l|}
\hline Ankle (Supine Position) & T1 FSE & STIR & PD & T2 SPAIR & PD \\
\hline Plane & Sagittal & Sagittal & Coronal & Axial & Axial \\
\hline TR (ms) & $700-1000$ & $2000-4000$ & $2000-4000$ & $2000-4000$ & $2000-4000$ \\
\hline TE (ms) & 10 & 30 & 30 & 60 & 38 \\
\hline TI (ms) & & 190 & & 190 & \\
\hline FOV (mm) & $160 \times 160$ & $160 \times 160$ & $140 \times 140$ & $140 \times 140$ & $140 \times 140$ \\
\hline Matrix size (1.5T) & $389 \times 456$ & $272 \times 400$ & $432 \times 333$ & $363 \times 400$ & $368 \times 400$ \\
\hline Thickness (mm)/Skip & $3 / 0$ & $3 / 0$ & $2.5 / 0$ & $3 / 0$ & $3 / 0$ \\
\hline NEX & 1 & 2 & 1 & 2 & 1 \\
\hline Turbo factor/ETL & 3 & 5 & 6 & 7 & 9 \\
\hline Bandwidth (Hz/pixel) & 440 & 401 & 440 & 401 & 440 \\
\hline Forefoot (Supine Position) & T1 FSE & T2 SPAIR & PD & T1 FSE & T2 SPAIR \\
\hline Plane & Sagittal & Sagittal & Coronal & Axial & Axial \\
\hline TR (ms) & $700-1000$ & $2000-4000$ & $2000-4000$ & $500-1000$ & $2000-4000$ \\
\hline TE (ms) & 10 & 60 & 37 & 10 & 60 \\
\hline FOV (mm) & $150 \times 150$ & $260 \times 260$ & $140 \times 140$ & $140 \times 140$ & $140 \times 140$ \\
\hline Matrix size (1.5T) & $418 \times 500$ & $323 \times 376$ & $396 \times 468$ & $367 \times 400$ & $352 \times 308$ \\
\hline Thickness (mm) & $2.5 / 0$ & $2.5 / 0$ & $2.5 / 0$ & $2.5 / 0$ & $2.5 / 0$ \\
\hline NEX & 1 & 2 & 1 & 2 & 1 \\
\hline Turbo factor/ETL & 3 & 8 & 6 & 7 & 8 \\
\hline Bandwidth (Hz/pixel) & 440 & 400 & 440 & 440 & 400 \\
\hline
\end{tabular}

FSE, fast spin echo; STIR, short tau inversion recovery; FS, frequency-selective fat saturated; PD, proton density; FOV, field of view; NEX, number of excitations; ETL, echo train length; BW, bandwidth.

T2 FS is usually used when imaging the forefoot rather than STIR unless significant artifacts result.

Although the simultaneous addition of a gallium scan may produce a modest increase in accuracy, the addition of labeled white blood cell scan (e.g., Indium-111-labeled leucocytes) to detect neutrophil accumulation increases the sensitivity, specificity, and accuracy to $>90 \%{ }^{22}$ At our institution we routinely add a sulfur colloid scan as a marrow map in patients with prior orthopedic instrumentation.

Although ${ }^{18} \mathrm{~F}$-fluorodeoxyglcose positron emission imaging (FDG-PET) has emerged as a sensitive modality in the detection and management of multiple inflammatory and infectious conditions, no role has been described in the diagnosis of AVN. Presently FDG-PET lacks sufficient specificity to be clinically useful in the imaging of postoperative complications of joint prostheses. ${ }^{23}$

\section{Ankle and Hindfoot AVN}

\section{Distal Tibia}

AVN of the distal tibia post ankle fracture in the adult is uncommon. An isolated case report after Maisonneuve-type fracture of the proximal third of the fibula with syndesmotic injury treated with lateral K-wire fixation of the syndesmosis and ligament reconstruction developed symptomatic and radiographic evidence of AVN of the tibial plafond 7 months after injury. ${ }^{24}$ One published clinical series of nine patients, each of whom sustained a Weber C-type fracture-dislocation of the tibio-talar joint, developed plain radiographic evidence of AVN of the lateral tibial plafond between 4 and 8 months postinjury. ${ }^{25}$ An unpublished series of nine patients by Linklater (personal correspondence) demonstrated the coincidence of syndesmotic injury and distal tibial metaphyseal or plafond bone infarction. All nine cases had MR features of syndesmotic injury, and five of these cases were initially treated with lateral syndesmotic screw fixation.

In our recent experience, we have observed AVN of the distal tibia following Maisonneuve-type proximal fibular fracture with transsyndesmotic screw fixation (-Fig. 4), Weber C fibular fracture with associated posterior malleolar fracture, and Weber $C$ trimalleolar fractures treated with internal fixation without syndesmotic screw fixation. A common observation in our cases and in those from the literature is AVN consistently involving the posterolateral tibial plafond after injuries to the distal tibio-fibular syndesmosis with concomitant suprasyndesmotic fibula fracture.

Posttraumatic AVN of the distal tibia is probably multifactorial and related to the relatively tenuous blood supply of the lateral tibial plafond, the mechanism of injury, and the treatment methods used. The distal tibial blood supply arises from nutrient arteries from the posterior tibial artery (PTA) and extraosseous metaphyseal and periosteal arteries from branches of the PTA, anterior tibial artery (ATA), and peroneal artery (PA) with a prominent medial 

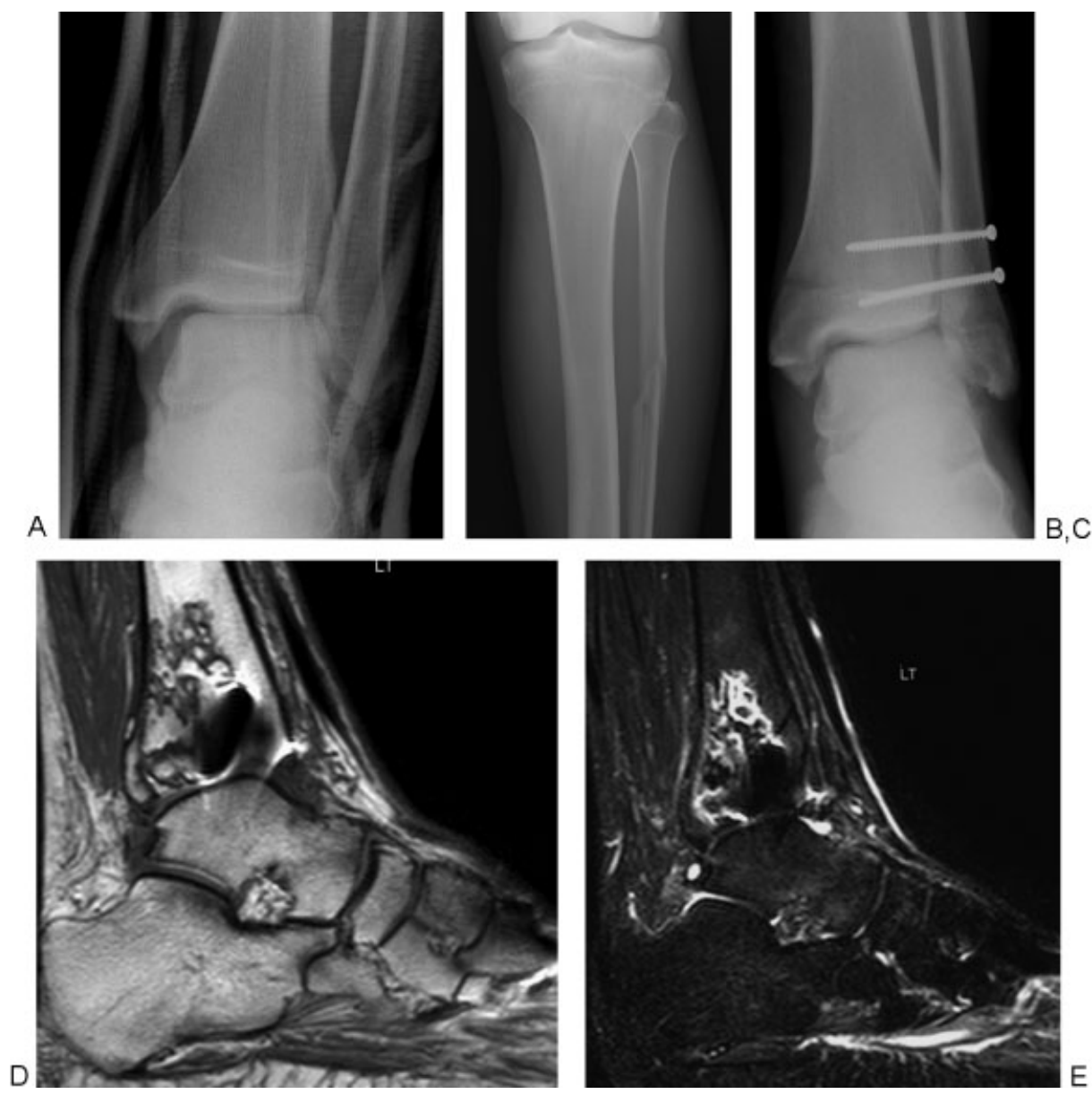

Figure 4 Avascular necrosis (AVN) of the distal tibial metaphysis and plafond in elite basketball player after Maisonneuve-type fibula fracture with syndesmotic injury. (A, B) Anteroposterior (AP) radiograph of the ankle shows widening of the medial and lateral clear space and loss of congruity of the distal tibiofibular syndesmosis. AP radiograph of the proximal fibula confirms Maisonneuve-type fracture. (C) Initial postoperative AP radiograph demonstrates reduction of the distal syndesmosis with two transsyndesmotic screws. (D, E) Coronal T1 fast spin-echo and short tau inversion recovery MRI, demonstrating AVN in the posterolateral distal tibia with disruption of the articular surface. Note the susceptibility artifact associated with transsyndesmotic screws.

perimalleolar anastomotic ring. ${ }^{25-27}$ The distal lateral tibial epiphysis is relatively less vascularized compared with the medial and is primarily supplied by the ATA and PA. ATA branches alone nourish this region in approximately a third of cases, possibly further predisposing to ischemia. $^{25}$

The association in case reports with syndesmotic injury, bimalleolar and trimalleolar fractures, and tibio-talar joint dislocation suggests patients at risk of AVN have an unstable injury. Initial radiographic findings suggestive of such injuries include reduced tibiofibular overlap (syndesmosis widening), concomitant posterior malleolar fracture, and Weber $\mathrm{C}$ fracture or Maisonneuve fracture, tibio-talar joint dislocation, marked soft tissue swelling, and subcutaneous gas associated with open fracture. CT, MRI, and stress radiographs are more sensitive than plain radiographs for syndesmosis diastasis. MRI determines the extent of interosseous membrane tearing more accurately than estimation from the level of fibula fracture. ${ }^{28}$ In cases likely predisposed to AVN, advanced imaging is not routinely performed preoperatively because the presence of posterior malleolar or suprasyndesmotic fibula fractures necessitates intraoperative surgical assessment of syndesmotic stability.

Surgical techniques aim for the anatomical restoration of the disrupted distal tibiofibular syndesmosis and may require fibular fracture internal fixation, repair of medial malleolar fracture/ligamentous injury, posterior malleolar fracture fixation (especially if large and posterior inferior tibiofibular ligament is attached), and transsyndesmotic screw fixation. ${ }^{28,29}$ The perforating branch of the PA has been shown to be at risk with syndesmotic screw fixation where it traverses the interosseous membrane from posterior to anterior. ${ }^{30}$ Open plating of fractures of the distal tibial metaphysis has also been demonstrated to cause greater disruption of extraosseous blood supply than percutaneously applied plates. ${ }^{27}$ The hypothesis that operative disruption of the extraosseous blood supply of the distal tibia may contribute to AVN seems reasonable but has not been confirmed.

Distinguishing early radiographic signs of AVN from patchy sclerosis associated with fracture healing and hardware fixation can be challenging; however, temporal changes 


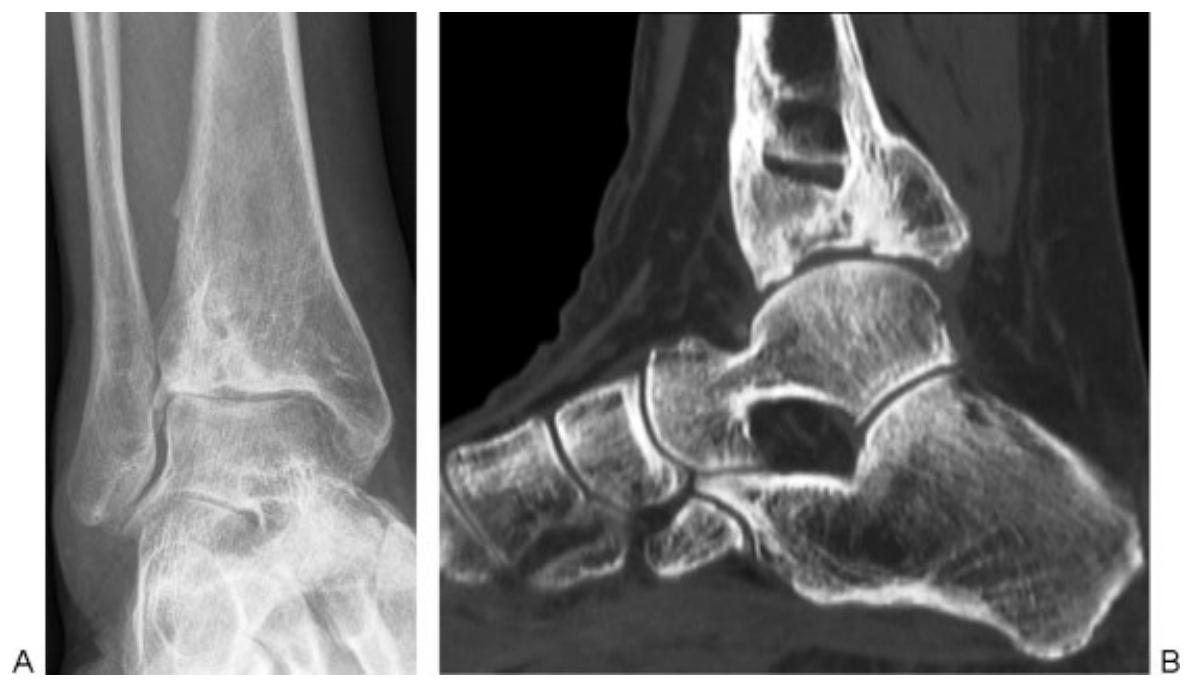

Figure 5 Avascular necrosis (AVN) of the distal tibial metaphysis detected 6 months after trimalleolar fracture in patient with ongoing pain after removal of orthopedic hardware. (A) Oblique radiograph of the right ankle with patchy sclerosis in the distal tibial metaphysis and plafond with subchondral "crescent sign." (B) Noncontrast computed tomography of the ankle sagittal reformat confirms healed posterior malleolar fracture, mixed hypoattenuation, and sclerosis in the distal tibial metaphysis, subchondral lucency, and partial collapse of the articular surface.

in the density of the distal metaphysis and plafond are usually present. With later stages of AVN progressive collapse of the tibial plafond, joint space narrowing and ankle joint deformity are seen with secondary osteoarthritis. Failure of previous surgery may predispose to subsequent AVN, and signs of hardware failure or residual instability of the syndesmosis should be sought (-Fig. 5). In such cases, CT is sensitive to detect plate and screw fracture and widening of the syndesmosis. Residual thickening and edema in the inferior syndesmotic ligaments and subtle marginated regions of AVN, depicted as the double-line sign, may be seen on MRI. CT and MRI also provide important complementary information on the integrity of the articular surface and extent of bone infarction for planning subsequent surgical salvage procedures including tibiotalar joint arthrodesis or total ankle arthroplasty with or without bone grafting.

\section{Talus}

AVN of the talus is common, in part due to its susceptibility to trauma with high-energy dorsiflexion, and partly due to its reliance on direct vasculature for its blood supply. There are no tendinous attachments or muscle origins and $\sim 60 \%$ of the talar surface is covered by articular cartilage, leaving little area for vascular perforation. ${ }^{3,7}$

Major extraosseous arterial supply comes from branches of the posterior tibial artery, anterior tibial artery, dorsalis pedis artery, and perforating peroneal artery with a key anastomosis in the sinus tarsi between the artery of the tarsal canal and tarsal sinus artery.

There is a significant retrograde component of the intraosseous blood supply of the talus that is important in the trauma setting because patients with a fracture of the talar neck usually develop AVN in the talar body. The Hawkins classification of talar neck fractures, later revised by Canale and Kelly, recognizes the increased likelihood of developing AVN with increased fracture displacement and the presence of associated dislocations. Type IV fractures, involving dislocation or subluxation of the subtalar, tibiotalar, and talonavicular joints, almost universally cause AVN. ${ }^{10,31}$ The risk of $A V N$ increases in talar neck fractures that extend to involve the body, increasingly comminuted and displaced talar body fractures, and with talar head fractures. ${ }^{3,32,33}$ A commonality of higher grade injuries is the increased disruption to the extraosseous blood supply and soft tissue attachments that occurs with dislocations.

AVN of the talus has been described in cases reports of surgical procedures around the ankle and foot including medial and lateral release of congenital clubfoot, tibio-talarcalcaneal arthrodesis, triple arthrodesis, and talonavicular arthrodesis (-Fig. 6). ${ }^{34,35}$ A triple arthrodesis, often performed via a lateral approach with anterior to posterior technique, may disrupt the tarsal sinus artery when the chondral surfaces are removed. This necessitates reliance on intraosseous anastomoses with the artery of the tarsal canal and fused osseous surfaces to supply the lateral aspect of the talar dome. ${ }^{34}$ The more extensively the talar body and neck are resected, the more likely is subsequent AVN. The medial approach used for talonavicular arthrodesis may disrupt the artery of the tarsal canal or intraosseous anastomoses and may also result in AVN of the lateral talar body and dome. ${ }^{35}$

Detection of the Hawkins sign 4 to 8 weeks after fracture or surgical intervention is suggestive of revascularization of the relevant portion of the talar body, and it usually progresses from medial to lateral. A partial Hawkins sign indicative of incomplete AVN is more commonly observed in the medial talus, indicating susceptibility to AVN of the lateral talar dome or inferior articular surface of the body. ${ }^{7}$ Complete revascularization after surgery may take between 6 months and 3 years. During this time fractures may heal as progressive sclerosis and cystic changes of AVN either resolve or lead to osseous collapse. MRI performed $>3$ weeks after surgery is more sensitive than plain radiographs in the 

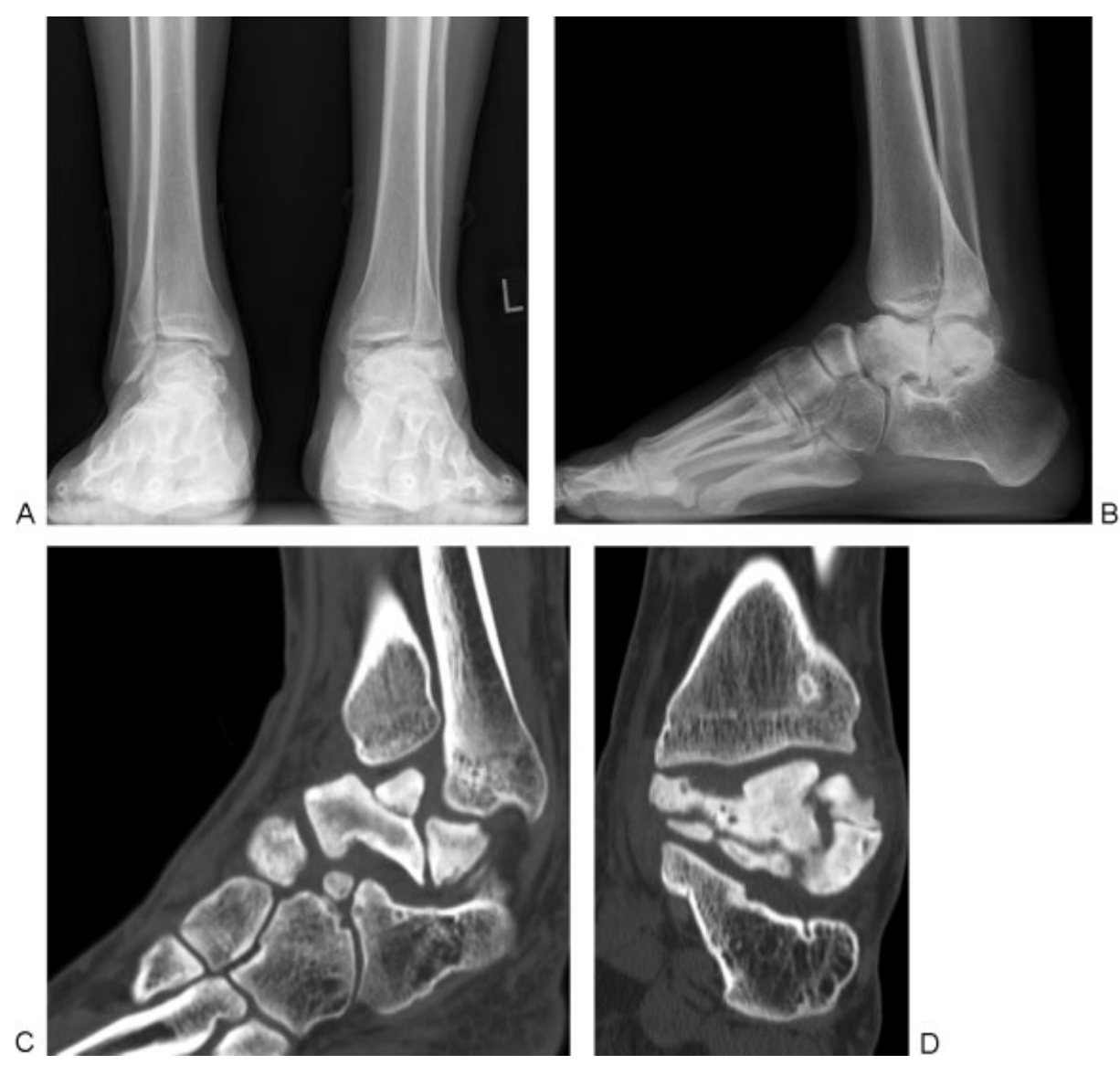

Figure 6 Sclerosis of tripartite talus secondary to medial clubfoot release, congenital talar dysplasia, subchondral avascular necrosis, and chronic nonunion. (A, B) Anteroposterior weightbearing radiographs of both ankles and lateral radiograph left ankle. (C, D) Noncontrast computed tomography with sagittal and coronal reformats demonstrates dense sclerosis and corticated chronic nonunion of talar fragments.

assessment of AVN in Hawkins type II and type III fractures, and it provides important prognostic information regarding the volume of avascular bone and risk of subsequent collapse (-Fig. 7). ${ }^{36} \mathrm{CT}$ is particularly useful in the assessment of healing or evolution of AVN after triple arthrodesis ( - Fig. 8).
Imaging findings are key determinants in the suitability for conservative management, core decompression, or bone graft procedures. Salvage arthrodesis or resection is usually required after significant collapse and secondary osteoarthritis supervenes.
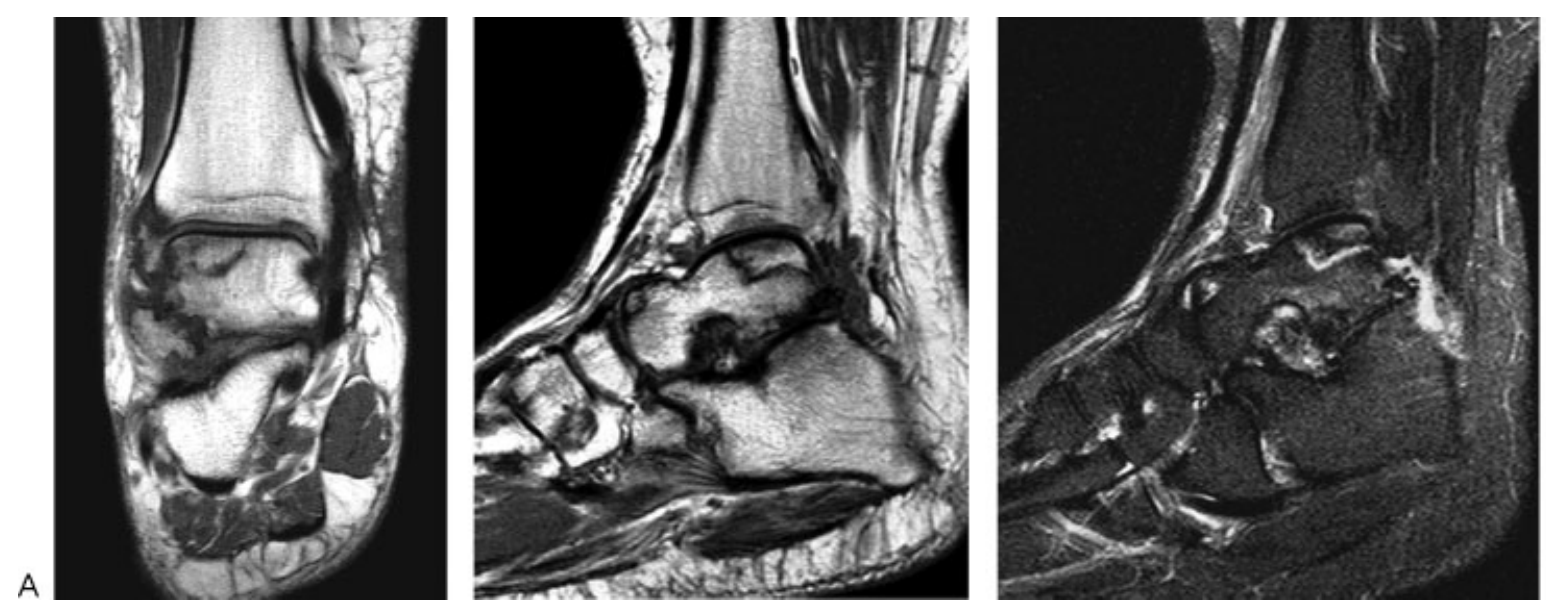

$B, C$

Figure 7 Lateral talar body/dome avascular necrosis (AVN) after surgical reduction of Hawkins type III fracture of the talar neck/body with dislocation of the talar body from the subtalar and ankle joints. Focal subchondral AVN also developed in the talar head. (A-C) Coronal T1 fast spin-echo (FSE), sagittal T1 FSE, and short tau inversion recovery MRI, demonstrating demarcated regions of AVN in the lateral talar body/dome and talar head with fracture extension into the lateral process and subtalar joint. 


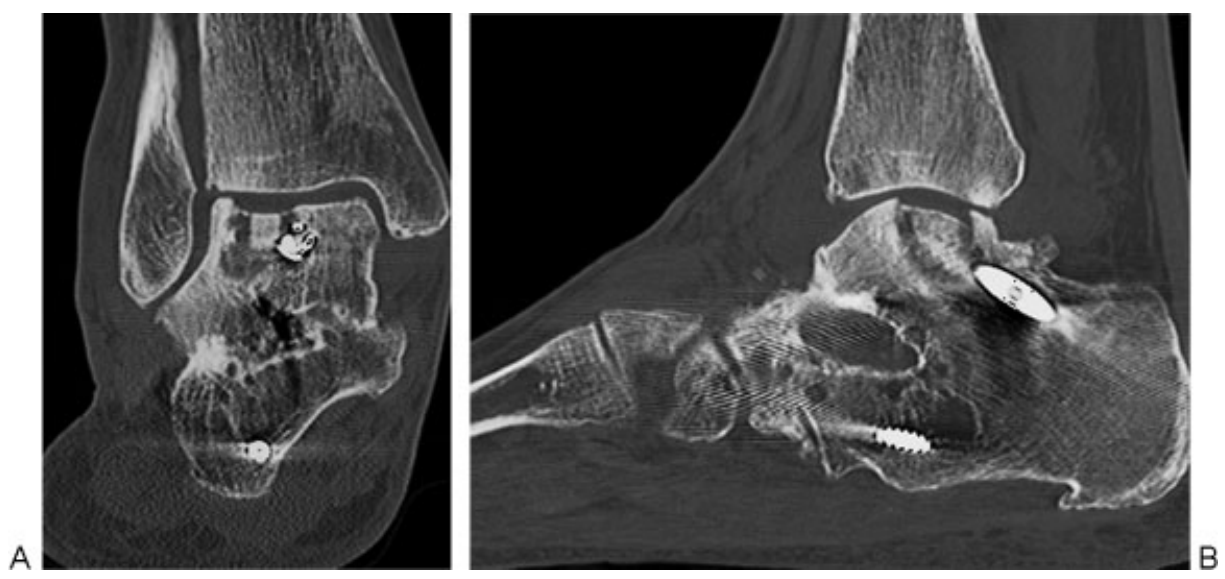

Figure 8 Avascular necrosis of the lateral talar dome detected 8 months after triple hindfoot arthrodesis for symptomatic calcaneonavicular fibrous coalition, pes planus, and posterior tibialis tendon dysfunction. Infection was excluded clinically and with nuclear scintigraphy.

(A, B) Noncontrast computed tomography with coronal and sagittal reformats demonstrates sclerosis and subchondral fracture of the lateral talar dome with subchondral collapse.

\section{Midfoot and Forefoot}

\section{Navicular}

The navicular is vascularized dorsally from a branch of the dorsalis pedis artery with the plantar surface supplied by the medial plantar artery, entering through the nonarticular surface. The intraosseous flow is centripetal with the center of the bone susceptible to AVN. ${ }^{3,37}$ Trauma is the most common etiology with injuries ranging from avulsion frac- tures to comminuted fracture-dislocations. Fractures of the body, usually due to high energy trauma including motor vehicle accidents, are the most likely to develop AVN and are classified according to the Sangeorzan system. ${ }^{38}$ Type 1 fractures involve the primary fracture line transverse in the coronal plane, and type 3 fractures produce comminution in the middle and lateral navicular with dislocation of the naviculocuneiform joint. These two fracture types cause the greatest disruption to the radial blood supply and commonly

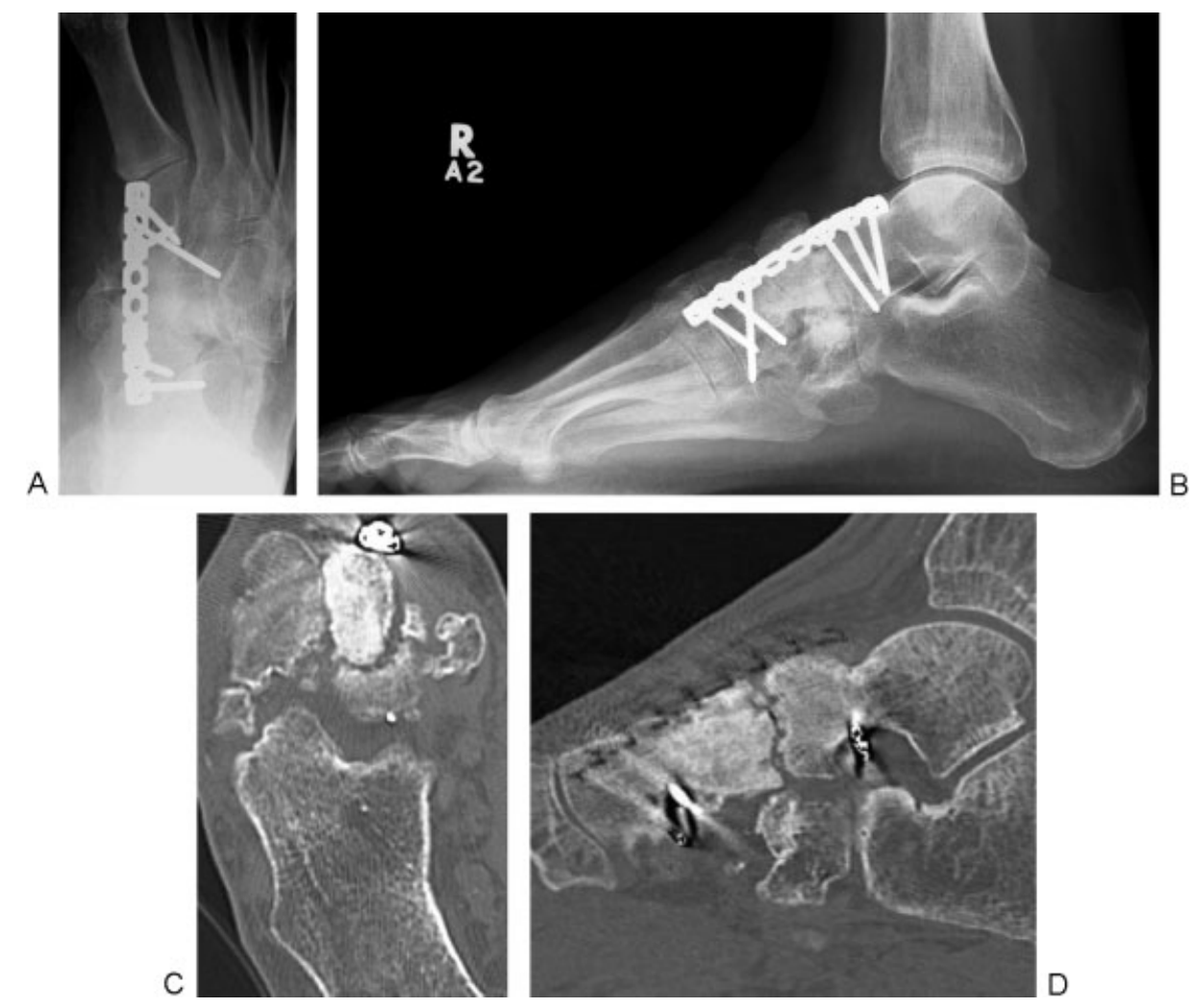

Figure 9 Nonunion of navicular allograft implanted after crush injury and type C comminuted fracture of navicular body. (A, B) Anteroposterior and lateral radiographs of the foot after buttress plate and screw fixation of navicular fracture with sclerosis and partial collapse mimicking avascular necrosis. (C, D) Noncontrast computed tomography scan with axial and sagittal reformats demonstrates sclerosis in the allograft with fragmentation and collapse of the native navicular. The patient proceeded to triple arthrodesis. 

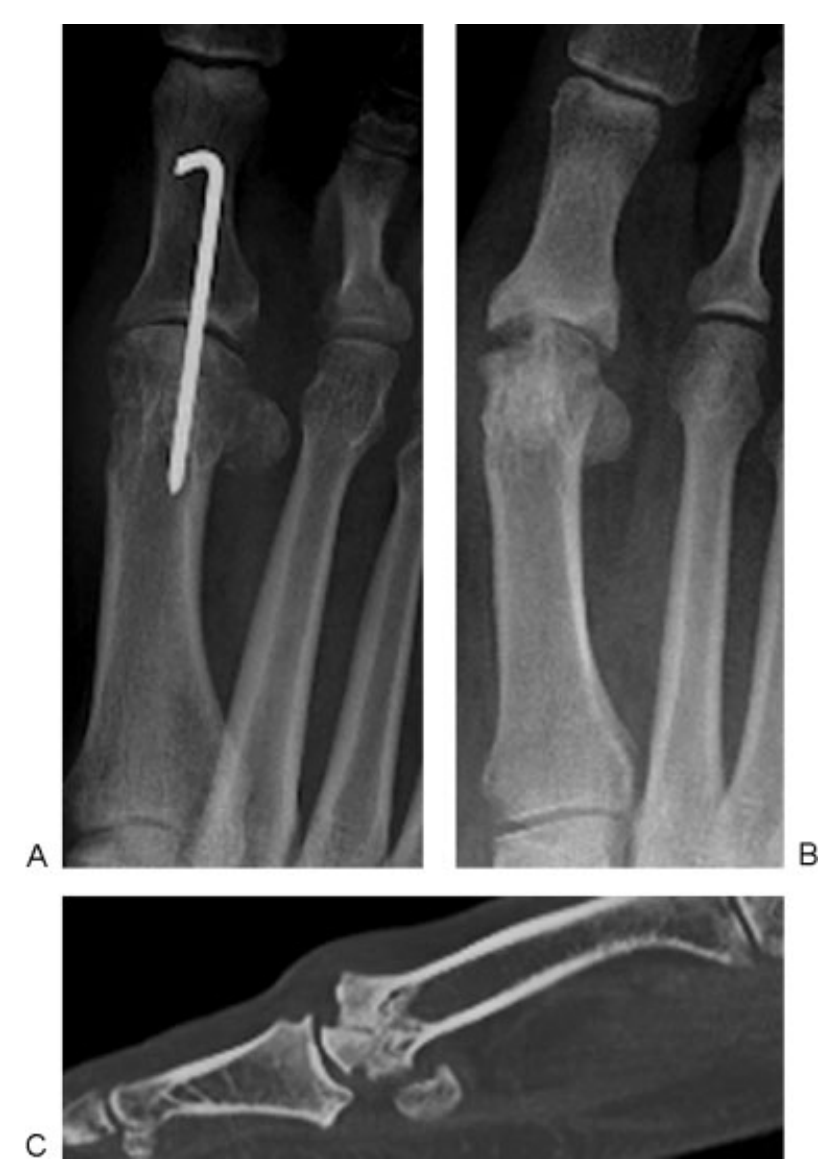

Figure 10 Avascular necrosis (AVN) of first metatarsal after Chevron osteotomy and lateral release. (A) Anteroposterior (AP) radiograph 4 weeks postoperative with K-wire fixation. (B) AP radiograph 8 months postoperative with fragmentation and collapse of the metatarsal head articular surface. (C) Noncontrast computed tomography scan with sagittal reformats confirms union of the Chevron osteotomy with AVN and collapse of the first metatarsal head.

result in AVN with a probable contribution from soft tissue stripping at the time of injury or surgical exposure. ${ }^{3,37}$

Severely comminuted fractures with dislocation may initially be treated with external fixation with eventual definitive fixation with a buttress plate to restore navicular length and naviculocuneiform screw fixation with or without bone grafting. ${ }^{39} \mathrm{CT}$ is the appropriate imaging modality to assess for fracture healing, bone graft incorporation, and complications including AVN. Autografts from the calcaneus, medial malleolus, or iliac crest and bulk allograft have attenuation similar to that of adjacent cortical bone on CT. Allografts (cadaveric bone transplants) may be in the form of bone chips or morsels that are hyperdense relative to adjacent bone. ${ }^{40}$ Immediate postoperative radiographs or CT easily distinguish graft material from native bone and are useful as a reference landmark of graft volume. Delayed complications of bone grafts including nonunion, delayed union, and graft extrusion may mimic AVN as they are depicted radiographically as sclerosis, erosion, and fragmentation of the graft with persistent lucency at the graft-host junction (- Fig. 9). ${ }^{40}$

\section{First Metatarsal}

First metatarsal AVN primarily affects the head and occurs most commonly after hallux valgus correction surgery. The chevron osteotomy, the most commonly performed realignment procedure, consists of a $\mathrm{V}$-shaped distal osteotomy that allows the first metatarsal head to be shifted laterally. Reported rates of $A V N$ for this procedure are between $0 \%$ and $20 \%$ with similar rates for Keller's arthroplasty of the first metatarsophalangeal joint. ${ }^{41,42} \mathrm{AVN}$ is also a rare complication of isolated cheilectomy for repair of hallux rigidus. ${ }^{43}$ Diaphyseal osteotomies, such as the Scarf procedure, and base or proximal approaches, such as the Lapidus procedure (involving capsular release, cheilectomy, and fusion of the first tarsometatarsal joint), have lower rates of AVN.

The first metatarsal head is supplied by branches of dorsalis pedis and posterior tibial arteries, including the first dorsal metatarsal, first plantar metatarsal, and superficial medial plantar arteries. ${ }^{41}$ The contribution to blood supply of the various branches is debated; however, a pericapsular anastomosing plexus from these vessels has been described on the plantar and lateral aspect of the metatarsal neck, branches of which supply the metatarsal head. ${ }^{3,41,42}$

The location of the osteotomy and extent of soft tissue resection may contribute to the development of AVN. ${ }^{3}$ The plantar limb of the chevron osteotomy has been demonstrated to emerge through the pericapsular anastomosis if the lateral cortex is breached. ${ }^{41}$ Additionally, a short dorsal limb may disrupt nutrient artery blood supply that arises from the dorsalis pedis branches and enters the lateral aspect of the distal shaft. ${ }^{44}$ Excessive capsular stripping for lateral release may also disrupt the lateral metaphyseal blood supply. ${ }^{45}$

Postoperative AVN of the first metatarsal may be asymptomatic or present with medial forefoot pain. Distinction of radiographic signs of AVN from other processes, including osteotomy and bone resection, thermal damage, hyperemia, altered mechanics, and osteotomy instability is difficult. Increased radiodensity, mild osseous resorption, and small subchondral cyst formation may be seen as part of the normal healing process following first metatarsal osteotomy. Sequential evolving lucency in the subchondral bone and eventual collapse are highly suggestive of AVN (-Fig. 10). CT is commonly used as a problem-solving modality in our practice to assess for osteotomy complications including malunion in dorsiflexion, nonunion, and AVN. Small field of view MRI is ideally suited to imaging cartilage due to the excellent resolution and contrast discrimination, assisting in the distinction of AVN of the metatarsal from osteoarthritis. ${ }^{46}$

\section{Lesser Metatarsals}

Bone marrow edema syndrome, detected on MRI in the lesser metatarsals as a rare cause of metatarsalgia after chevron osteotomy, is thought to be related to stress or friction-related pathology associated with altered biomechanics. ${ }^{47}$ Whether this represents an early phase of metatarsal head subchondral fracture or AVN is unclear because a severe bone marrow edema-like pattern is also seen in the lesser metatarsal heads in the early phases of infraction, followed later by head flattening, linear subchondral T1 signal, and arthrosis. ${ }^{48}$ 


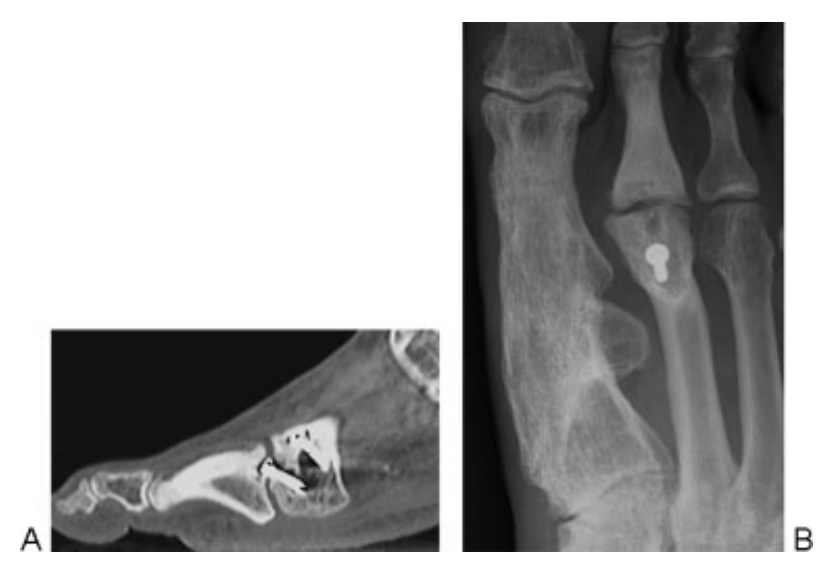

Figure 11 Avascular necrosis (AVN) of second metatarsal head after bone block fusion of the first metatarsal and Weil osteotomy second metatarsal. (A) Noncontrast computed tomography (CT) scan with sagittal reformats 4 years after initial operation demonstrates sclerosis and partial collapse of the dorsal second metatarsal head with the distal screw sitting proud of the metatarsal head in an intra-articular location. (B) Anteroposterior (AP) radiograph 1 year after the CT after removal of the distal screw with residual flattening and collapse of the second metatarsal head, consistent with previous AVN.

The blood supply of the lesser metatarsal heads arises from the dorsal metatarsal arteries (branches of the dorsalis pedis artery) and plantar metatarsal arteries (from the posterior tibial artery). ${ }^{3}$ A nutrient artery traverses the cortex of the distal metaphysis laterally (in the second to fourth metatarsals) and medially (in the fifth) close to capsular and ligamentous attachments, with terminal distal branches contributing to the blood supply of the metatarsal head. ${ }^{49}$ An extraosseous arterial anastomotic ring from branches of the dorsal and plantar metatarsal arteries forms around the metatarsal heads, which may be affected by metatarsal head osteotomy or extensive capsular stripping.

Chevron, Helal, or Weil osteotomies of the lesser metatarsals may be performed to correct chronic metatarsophalangeal joint dislocation, metatarsalgia due to an excessively long metatarsal, or intractable callosities. The most commonly performed procedure, the Weil osteotomy, consisting of an oblique osteotomy through the metatarsal head and shaft parallel to the ground with detachment of lateral capsular ligaments, provides controlled shortening of the metatarsal without increasing the load in the adjacent metatarsals. ${ }^{50}$ The direction of the osteotomy cuts places the intraosseous blood supply at risk in addition to the extraosseous capsular supply if the plantar cortex is breached. Recognized complications of the procedure include "floating toe" (a toe that does not contact the ground), nonunion, malunion, and transfer metatarsalgia. Although AVN is mentioned in the surgical literature as a potential complication, no case reports have been published in the English literature, and this is likely a very rare outcome. We have observed one case of probable AVN of the second metatarsal head after first metatarsal bone block fusion and Weil osteotomy of the second metatarsal, with the patient experiencing intractable second metatarsalgia 5 years after the original procedure (-Fig. 11).

\section{Conclusion}

The imaging modalities and major causes of postoperative AVN in the ankle and foot briefly discussed in this review represent those that radiologists are most likely to encounter in clinical practice.

Although rare, detection of AVN in the postoperative patient is critical because the diagnosis is often not suspected clinically, and appropriate management of these patients may be instituted to achieve satisfactory functional outcomes.

\section{References}

1 McCarthy I. The physiology of bone blood flow: a review. J Bone Joint Surg Am 2006;88(Suppl 3):4-9

2 Assouline-Dayan Y, Chang C, Greenspan A, Shoenfeld Y, Gershwin ME. Pathogenesis and natural history of osteonecrosis. Semin Arthritis Rheum 2002;32(2):94-124

3 DiGiovanni CW, Patel A, Calfee R, Nickisch F. Osteonecrosis in the foot. J Am Acad Orthop Surg 2007;15(4):208-217

4 Steffen RT, Athanasou NA, Gill HS, Murray DW. Avascular necrosis associated with fracture of femoral head after hip resurfacing. J Bone Joint Surg Br 2010;92(6):787-793

5 Bartonícek J, Fric V, Skála-Rosenbaum J, Dousa P. Avascular necrosis of the femoral head in pertrochanteric fractures: a report of 8 cases and a review of the literature. J Orthop Trauma 2007; 21(4):229-236

6 Pape D, Seil R, Anagnostakos K, Kohn D. Postarthroscopic osteonecrosis of the knee. Arthroscopy 2007;23(4):428-438

7 Pearce DH, Mongiardi CN, Fornasier VL, Daniels TR. Avascular necrosis of the talus: a pictorial essay. Radiographics 2005; 25(2):399-410

8 Koulouris G, Morrison WB. Foot and ankle disorders: radiographic signs. Semin Roentgenol 2005;40(4):358-379

9 Lafforgue P. Pathophysiology and natural history of avascular necrosis of bone. Joint Bone Spine 2006;73(5):500-507

10 Hawkins LG. Fractures of the neck of the talus. J Bone Joint Surg Am 1970;52(5):991-1002

11 Rammelt S, Zwipp H. Talar neck and body fractures. Injury 2009; 40(2):120-135

12 Resnick D, Sweet DE, Madewell JE. Osteonecrosis: pathogenesis, diagnostic techniques, specific situations, and complications. In: Diagnosis of bone and joint disorders. 4th ed. Philadelphia, PA: Saunders; 2002:3599-3685

13 Buckwalter KA, Parr JA, Choplin RH, Capello WN. Multichannel CT imaging of orthopedic hardware and implants. Semin Musculoskelet Radiol 2006;10(1):86-97

14 Lee MJ, Kim S, Lee SA, et al. Overcoming artifacts from metallic orthopedic implants at high-field-strength MR imaging and multidetector CT. Radiographics 2007;27(3):791-803

15 O'Hare A, Shortt C, Napier N, Eustace SJ. Bone marrow edema: patterns and clinical implications. Semin Musculoskelet Radiol 2006;10(4):249-257

16 Gyftopoulos S, Bencardino JT. Normal variants and pitfalls in MR imaging of the ankle and foot. Magn Reson Imaging Clin N Am 2010;18(4):691-705

17 Elias I, Zoga AC, Schweitzer ME, Ballehr L, Morrison WB, Raikin SM. A specific bone marrow edema around the foot and ankle following trauma and immobilization therapy: pattern description and potential clinical relevance. Foot Ankle Int 2007; 28(4):463-471

18 Weishaupt D, Schweitzer ME. MR imaging of the foot and ankle: patterns of bone marrow signal abnormalities. Eur Radiol 2002; 12(2):416-426 
19 Hintermann B. What the orthopaedic foot and ankle surgeon wants to know from MR imaging. Semin Musculoskelet Radiol 2005;9(3):260-271

20 Mont MA, Ulrich SD, Seyler TM, et al. Bone scanning of limited value for diagnosis of symptomatic oligofocal and multifocal osteonecrosis. J Rheumatol 2008;35(8):1629-1634

21 Hain SF, Fogelman I. Nuclear medicine studies in metabolic bone disease. Semin Musculoskelet Radiol 2002;6(4):323-329

22 Palestro CJ, Love C, Bhargava KK. Labeled leukocyte imaging: current status and future directions. Q J Nucl Med Mol Imaging 2009;53(1):105-123

23 Love C, Tomas MB, Tronco GG, Palestro CJ. FDG PET of infection and inflammation. Radiographics 2005;25(5):1357-1368

24 Lagier R. Case report 552: Post-traumatic remodelling of the distal tibial epiphysis: a form of aseptic osteonecrosis. Skeletal Radiol 1989;18(4):331-333

25 Assal M, Sangeorzan BJ, Hansen ST. Post-traumatic osteonecrosis of the lateral tibial plafond. Foot Ankle Surg 2007;13:24-29

26 Nelson GE Jr, Kelly PJ, Peterson LF, Janes JM. Blood supply of the human tibia. J Bone Joint Surg Am 1960;42-A:625-636

27 Borrelli J Jr, Prickett W, Song E, Becker D, Ricci W. Extraosseous blood supply of the tibia and the effects of different plating techniques: a human cadaveric study. J Orthop Trauma 2002; 16(10):691-695

28 Zalavras C, Thordarson D. Ankle syndesmotic injury. J Am Acad Orthop Surg 2007;15(6):330-339

29 van den Bekerom MP, Hogervorst M, Bolhuis HW, van Dijk CN. Operative aspects of the syndesmotic screw: review of current concepts. Injury 2008;39(4):491-498

30 Fanter NJ, Inouye SE, McBryde AM Jr. Safety of ankle transsyndesmotic fixation. Foot Ankle Int 2010;31(5):433-440

31 Canale ST, Kelly FB Jr. Fractures of the neck of the talus. Long-term evaluation of seventy-one cases. J Bone Joint Surg Am 1978; 60(2):143-156

32 Inokuchi S, Ogawa K, Usami N. Classification of fractures of the talus: clear differentiation between neck and body fractures. Foot Ankle Int 1996;17(12):748-750

33 Rammelt S, Zwipp H. Talar neck and body fractures. Injury 2009;40 (2):120-135

34 Jones CK, Nunley JA. Osteonecrosis of the lateral aspect of the talar dome after triple arthrodesis. A report of three cases. J Bone Joint Surg Am 1999;81(8):1165-1169

35 Hermus JPS. Osteonecrosis of the talus after talonavicular arthrodesis: a case report and review of the literature. J Foot Ankle Surg 2011;50(3):343-346
36 Thordarson DB, Triffon MJ, Terk MR. Magnetic resonance imaging to detect avascular necrosis after open reduction and internal fixation of talar neck fractures. Foot Ankle Int 1996;17(12): 742-747

37 Sizensky JA, Marks RM. Imaging of the navicular. Foot Ankle Clin 2004;9(1):181-209

38 Sangeorzan BJ, Benirschke SK, Mosca V, Mayo KA, Hansen ST Jr. Displaced intra-articular fractures of the tarsal navicular. J Bone Joint Surg Am 1989;71(10):1504-1510

39 DiGiovanni CW. Fractures of the navicular. Foot Ankle Clin 2004; 9(1):25-63

40 Beaman FD, Bancroft LW, Peterson JJ, Kransdorf MJ, Menke DM, DeOrio JK. Imaging characteristics of bone graft materials. Radiographics 2006;26(2):373-388

41 Malal JJG, Shaw-Dunn J, Kumar CS. Blood supply to the first metatarsal head and vessels at risk with a chevron osteotomy. J Bone Joint Surg Am 2007;89(9):2018-2022

42 Prasad MG, Shankar NS. Clinical results of Keller's arthroplasty. Foot 1998;8:223-225

43 Brosky TA II, Menke CRD, Xenos D. Reconstruction of the first metatarsophalangeal joint following post-cheilectomy avascular necrosis of the first metatarsal head: a case report. J Foot Ankle Surg 2009;48(1):61-69

44 Weinraub GM, Meberg R, Steinberg JS. Vascular perfusion of the long dorsal arm versus chevron osteotomy: a cadaveric injection study. J Foot Ankle Surg 2004;43(4):221-224

45 Jones KJ, Feiwell LA, Freedman EL, Cracchiolo A III. The effect of chevron osteotomy with lateral capsular release on the blood supply to the first metatarsal head. J Bone Joint Surg Am 1995; 77(2):197-204

46 Shortt CP. Magnetic resonance imaging of the midfoot and forefoot: normal variants and pitfalls. Magn Reson Imaging Clin N Am 2010;18(4):707-715

47 Aigner N, Petje G, Steinboeck G, Schneider W, Krasny C, Landsiedl F. Bone marrow edema of the forefoot after chevron osteotomy-a rare cause of metatarsalgia: a case report. Foot Ankle Int 2002; 23(5):447-451

48 Torriani M, Thomas BJ, Bredella MA, Ouellette H. MRI of metatarsal head subchondral fractures in patients with forefoot pain. AJR Am J Roentgenol 2008;190(3):570-575

49 Petersen WJ, Lankes JM, Paulsen F, Hassenpflug J. The arterial supply of the lesser metatarsal heads: a vascular injection study in human cadavers. Foot Ankle Int 2002;23(6):491-495

50 Espinosa N, Maceira E, Myerson MS. Current concept review: metatarsalgia. Foot Ankle Int 2008;29(8):871-879 